\title{
Nutrient availability modulates both host and parasite life histories in a snail-trematode interaction
}

\author{
Gregory J. Sandland*, Katherine A. Josephson
}

Department of Biology, University of Wisconsin-La Crosse, 1725 State Street, La Crosse, WI 54601, USA

\begin{abstract}
Host nutrient availability can have important life history consequences for hosts and their parasites. The responses of each participant within the association can vary extensively across host-parasite systems. In this paper, we outline the life history responses of the aquatic snail Biomphalaria glabrata and its trematode parasite Echinostoma caproni during host nutrient restriction. The onset of host starvation had rapid and strong effects on snail reproduction, significantly reducing egg output in control snails and eliminating egg production in infected individuals. The combination of E. caproni infection and nutrient restriction also had a dramatic effect on B. glabrata survival, with starved infected snails dying at a faster rate than hosts from any other treatment. In terms of parasite reproduction, host nutrient restriction did not influence the quantity of parasite larvae produced after starvation onset but did influence parasite quality, reducing both larval swimming time and overall longevity. Together these results demonstrate that nutrient restriction can strongly influence both host and parasite life histories, and therefore should be considered in future studies attempting to understand patterns of disease in host populations.
\end{abstract}

KEY WORDS: Biomphalaria glabrata $\cdot$ Echinostoma caproni $\cdot$ Starvation $\cdot$ Reproduction · Disease · Transmission · Survival

\section{INTRODUCTION}

Host nutrient availability is widely recognized as an important factor mediating infection outcomes across an array of host-parasite associations (Jokela et al. 2005, Tschirren et al. 2007, Muturi et al. 2011, Brodersen et al. 2012, Forbes et al. 2015) including those involving humans and their pathogens (Lefèvre et al. 2013). Recently, there has been increasing interest in this research area, as studies have suggested that host nutrient restriction not only impacts individual parasites, but parasite populations, and also influences the magnitude and spread of disease (Pedersen \& Greives 2008, Beldomenico \& Begon 2010, Tadiri et al. 2013, Civitello et al. 2015).

Investigations into nutrient limitation and its role in the infection process have often focused on host life history responses after infection has successfully occurred (Jokela et al. 2005, Rivero et al. 2007,
Brodersen et al. 2012). Less studied are the effects of limiting host resources on parasite establishment, development, and reproduction. Past work has revealed that nutrient limitation can result in increased susceptibility to parasitic infection (Sandland \& Minchella 2003a, Rondelaud et al. 2004, Beldomenico \& Begon 2010). Moreover, once established, parasites may be able to persist for longer periods of time and produce more offspring in (or on) hosts that have fewer resources to allocate towards anti-parasite defenses (Koski et al. 1999, Krasnov et al. 2005). Alternatively, nutrientdeprived hosts may constrain parasite reproduction simply through reduced resource availability (Ebert et al. 2004, Logan et al. 2005) and/or premature host (and therefore parasite) mortality resulting from the exhaustion of host resources (Jokela et al. 2005, Seppala et al. 2008). Parasite fitness may be further impacted if dispersal and spread are restricted by 
the limited mobility of energetically taxed hosts (Gerard 1996).

Parasite fitness can also be influenced by the quantity and/or quality of transmission stages. The degree to which parasites invest in each of these facets when exploiting nutrient-restricted hosts can have important consequences for transmission success and disease ecology (Dlugosz et al. 2014). In some studies, parasites infecting nutrient-deprived hosts have demonstrated reductions in offspring quantity and quality (Seppala et al. 2008). On the other hand, Krasnov et al. (2005) reported that fleas produced greater numbers of high-quality offspring when feeding on nutrient-deprived hosts compared to hosts fed nutrientrich diets. Together these studies not only highlight the variability observed across systems, but also emphasize the importance of investigating both quality and quantity endpoints to better understand how host nutrient deprivation potentially influences parasite transmission and subsequent disease spread.

Echinostoma caproni is a hermaphroditic flatworm parasite that requires 3 hosts to complete its life cycle. Adult worms reside in the intestinal tract of mammals or birds and release their eggs into the aquatic environment via host feces. Eggs eventually hatch, releasing miracidia which then infect the aquatic snail Biomphalaria glabrata as the first intermediate host. After weeks of intra-molluscan development and asexual amplification, cercariae are released from infected hosts. Cercariae then actively find suitable second intermediate hosts (including snails, fish, or amphibians) where they encyst as metacercariae (Toledo et al. 2007). The life cycle of this parasite is completed when mammals or birds consume second intermediate hosts harboring metacercariae. Once in the intestinal tract, metacercariae develop to adult worms within weeks of initial exposure and begin producing eggs, thereby completing the life cycle.

Substantial research on the E. caproni-B. glabrata system over the last 3 to 4 decades has led to its emergence as an important model for better understanding the complex relationships between hosts and their parasites (Beers et al. 1995, Bouchut et al. 2006, Toledo et al. 2007). Yet, to our knowledge no studies have investigated the role that nutrient restriction plays in modulating host and parasite life histories within this system. The goal of this study was to use this model host-parasite interaction to further expand our current understanding of the importance of host nutrient availability in disease emergence and spread by manipulating $B$. glabrata access to resources and subsequently monitoring both host and E. caproni life histories.

\section{MATERIALS AND METHODS}

\author{
Parasite material
}

Echinostoma caproni metacercariae were acquired from the laboratory of Dr. Tom Platt (Saint Mary's College, Indiana, USA) on 10 April 2012 and subsequently refrigerated at $4^{\circ} \mathrm{C}$ in well water. On 5 July 2012, metacercariae were fed to week-old leghorn chickens ( $\mathrm{n}=6$; Murray McMurray Hatchery, Iowa, USA) at a dose of 75 metacercariae chicken ${ }^{-1}$. At $16 \mathrm{~d}$ after inoculation, chickens were euthanized using $\mathrm{CO}_{2}$ followed by cervical dislocation (UW-La Crosse IACUC protocol 6-12). The intestine of each bird was removed and necropsied to acquire adult E. caproni worms, which were subsequently teased apart to obtain eggs. Parasite eggs were then transferred to a plastic petri plate containing well water and placed in the dark for $17 \mathrm{~d}$ at $22^{\circ} \mathrm{C}$. After that time, eggs were placed under incandescent light to induce miracidial hatching. These larvae were then used in snail exposures.

\section{Host material and exposures}

On 07 August 2012, Biomphalaria glabrata from our laboratory population at the University of Wisconsin-La Crosse were size-matched (5-9 mm) and allocated to either exposed $(\mathrm{n}=30)$ or control $(\mathrm{n}=$ 28) treatments. Snails were then individually added to $3 \mathrm{ml}$ plastic exposure wells with $2 \mathrm{ml}$ of water. B. glabrata in the exposed treatment received a dosage of 10 miracidia snail ${ }^{-1}$ whereas control snails received a sham exposure but were otherwise treated equally. All snails remained in these plastic wells overnight. The following day, snails were removed from their wells and placed individually into $150 \mathrm{ml}$ plastic cups where they were maintained for the remainder of the study. All snails were provided with lettuce ad libitum for the first $37 \mathrm{~d}$ of the study, and water was changed every 2 to $3 \mathrm{~d}$ throughout the entire study period. Styrofoam was also added to each cup to serve as a substrate for egg-mass deposition. Snails were measured weekly (widest diameter across the shell) using digital calipers, and the numbers of developing embryos within egg masses were counted twice each week. Styrofoam was scrapped after each count to provide a 'clean' substrate for subsequent snail reproduction. Snail survival was also assessed twice weekly. All dead snails were immediately necropsied to confirm infection status. 
At $38 \mathrm{~d}$ post exposure (DPE), snails from both control and infected treatments were further separated into either starved or fed groups. The onset of snail starvation was determined based on time to patency and our interest in acquiring a number of cercarial samples prior to starvation. Food was completely restricted in the starved treatment, whereas snails continued to receive lettuce ad libitum in the fed treatment. We included starvation as the nutrientrestricted treatment in our study for 2 reasons. First, freshwater snails have been reported to experience food limitation in stream habitats (Rosemond et al. 2000). Second, starvation has been incorporated into past research investigating interactions between host nutrient access and infection, which provided a more direct comparison between our results and those of others (Jokela et al. 2005, Seppala et al. 2008). This generated a $2 \times 2$ factorial design that included fedcontrol snails $(\mathrm{n}=14)$, starved-control snails $(\mathrm{n}=14)$, fed-infected snails $(\mathrm{n}=11)$, and starved-infected snails $(\mathrm{n}=9)$. The lower number of B. glabrata in each of the infected groups resulted from the fact that not all exposed snails actually reached patency. In the fed group, 11/15 individuals (73.3\%) reached patency, whereas $9 / 15$ snails $(60 \%)$ reached patency in the starved treatment. Estimates of egg production and snail growth continued for an additional $2 \mathrm{wk}$ after the onset of starvation treatments (which coincided with cercarial assessments), and host survival was followed for an additional 4 wk post starvation.

\section{Parasite collections and assessments}

Parasite quantity

To determine if parasite release differed between starved and fed hosts, parasites were collected from infected snails every 2 to $4 \mathrm{~d}$ between 26 and 47 DPE. This allowed us to capture the start of cercariae release and then follow cercariae output up to and after the onset of host starvation. To do this, equal numbers $(n=8)$ of size-matched infected snails from each nutritional treatment (starved vs. fed) were added individually to $10 \mathrm{ml}$ wells filled with well water. Wells were then placed under incandescent light for $4 \mathrm{~h}$ (13:00-17:00 h). After the first $2 \mathrm{~h}$ interval, a $1 \mathrm{ml}$ aliquot of cercarial suspension was extracted from each well and added to a standard petri plate. Ethanol (70\%) was then used to fix the parasites for subsequent enumeration using a dissecting microscope at $40 \times$ magnification. The remainder of the water in each well (along with any remaining cer- cariae) was then poured out and fresh well water was added. This process was repeated for the second $2 \mathrm{~h}$ shedding period. These numbers were then multiplied by 10 to get an overall estimate of parasite totals released by snails into the wells during each $2 \mathrm{~h}$ period. Totals from each $2 \mathrm{~h}$ shed were then summed to get an estimate of cercarial release snail ${ }^{-1}$ for each shedding event $(=4 \mathrm{~h})$.

\section{Parasite quality}

At 47 DPE (the final shedding day), cercariae were collected from a subset of size-matched infected snails $\left(\mathrm{n}=5\right.$ treatment $\left.^{-1}\right)$ after they had been under incandescent light for $1 \mathrm{~h}$. Five vigorous larvae were collected from each host, and each larva was placed individually into a $3 \mathrm{ml}$ plastic well with $2 \mathrm{ml}$ of well water. We chose to follow 5 cercariae snail ${ }^{-1}(=50$ cercariae in total), as this number allowed for replication within hosts while also allowing us to complete our swimming and crawling assessments within a relatively short time window. Cercariae were examined hourly, and 2 transmission metrics were determined at each time point: (1) whether or not the larvae were actively swimming (vs. crawling on the bottom) and (2) whether or not the cercariae were moving. We used these categories as indicators of transmission potential, as similar metrics have been used in past studies (Seppala et al. 2008) and also because echinostomes regularly use benthic snails as second intermediate hosts and therefore could come in contact with and attach to snails even while crawling. Larval monitoring continued each hour over a $24 \mathrm{~h}$ period.

\section{Data treatment and statistical analyses}

Snail size was compared using 2-way ANOVA where infection status, diet status, and their interaction were included in the model. This was done at 3 time points: Week 0 (day of exposure), Week 5 (prior to starvation), and Week 7 (post starvation). Egg totals were calculated prior to (Weeks 0-5) and then after (Weeks 6-7) starvation. Totals were then compared among treatments within each time block using 2-way ANOVA after square-root transformation. Again, infection status and diet status were included as main effects in the model along with their interaction. Because there was no mortality observed in snails from any of the treatment groups prior to starvation (Weeks 0-5), Kaplan-Meier sur- 
vivorship curve analysis was restricted to snails after diet manipulation (Weeks 6-9).

In order to compare cercarial release from fed versus starved snails over time, parasite numbers were first $\log _{10}$ transformed prior to repeated measures ANOVA. The first 2 shedding periods (04 and 08 September) were excluded from this analysis as none of the snails had reached patency. Mean proportions of swimming and surviving larvae were compared between fed and starved snails over time using repeated-measures ANOVA after arcsine squareroot transformations.

\section{RESULTS}

\section{Host parameters}

We used 2-way ANOVA to determine whether infection status and/or diet manipulation (plus their interaction) had any influence on snail size at 3 time points in the study: Week 0 (initial exposure), Week 5 (last week prior to starvation), and Week 7 (post starvation). Analyses revealed no effect of either factor (or the interaction) on snail size at any of the 3 time points (all $\mathrm{p}>0.05$ ). Mean snail sizes were within $1 \mathrm{~mm}$ of each other across treatments at each of the 3 time points.

Prior to diet manipulation, 2-way ANOVA revealed a significant influence of infection on egg output, with control snails producing over twice as many eggs as infected snails $\left(F_{1,44}=22.47, \mathrm{p}<0.001\right)$. Not surprisingly, both diet and the diet $x$ infection interaction were non-significant (all $p>0.05$ ) given that all snails were still receiving lettuce ad libitum. After the onset of food restriction, there was a significant decrease in the numbers of eggs generated by starved control snails compared to those that were fed $\left(t_{26}=3.66, p=0.001\right.$; Fig. 1). A starker reproductive pattern was seen in the infected snail groups, where fed-infected hosts continued to produce eggs (albeit at lower levels), whereas those from the starved treatment ceased reproducing entirely.

During the first $5 \mathrm{wk}$ of the study (pre-starvation), no mortality was observed in snails from any of the treatment groups. After diet manipulation, however, host mortality differed significantly among treatments $\left(\chi^{2}=54.69, \mathrm{p}<0.001\right)$, with infected snails exhibiting higher mortality than control snails (Fig. 2). Moreover, starved-infected snails demonstrated the highest mortality of any treatment, with only $11 \%$ of the individuals surviving at the end of the study (Fig. 2).

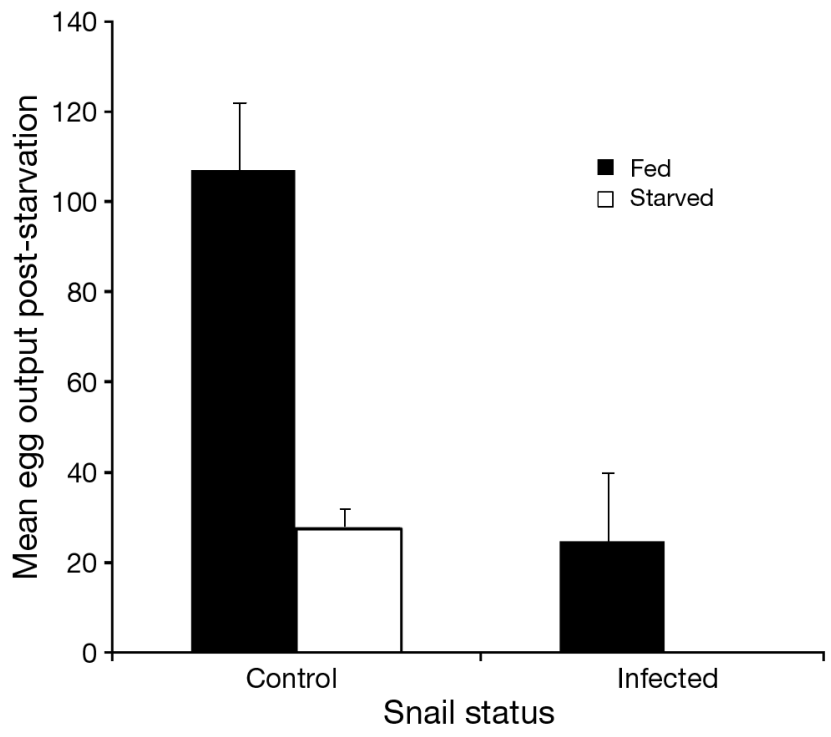

Fig. 1. Mean $( \pm \mathrm{SE})$ total number of eggs produced by Echinostoma caproni-infected and control Biomphalaria glabrata snails that were either starved or fed lettuce ad libitum for a 14 d period

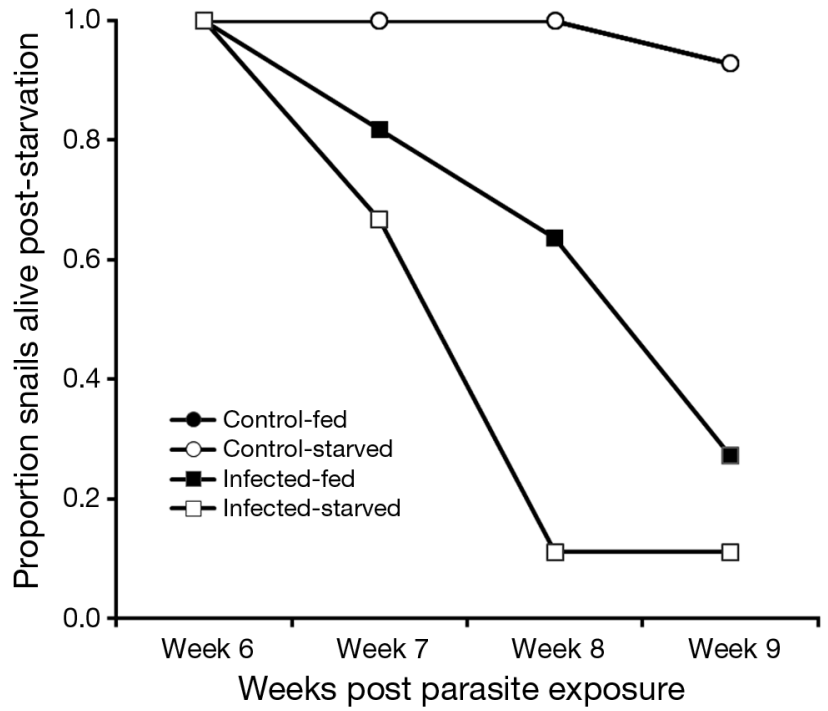

Fig. 2. Weekly survival of Echinostoma caproni-infected and control Biomphalaria glabrata snails that were either starved or fed lettuce ad libitum. Note that snails from the control-fed and control-starved treatments expressed the same survival pattern and therefore have overlapping lines

\section{Parasite parameters}

Repeated measures ANOVA revealed a significant effect of time on cercarial output $\left(F_{6,84}=16.65, \mathrm{p}<\right.$ 0.001), with parasite numbers generally increasing throughout the shedding period (Fig. 3). Neither diet nor the diet $\times$ time interaction was significant (all $\mathrm{p}>0.05$ ). 


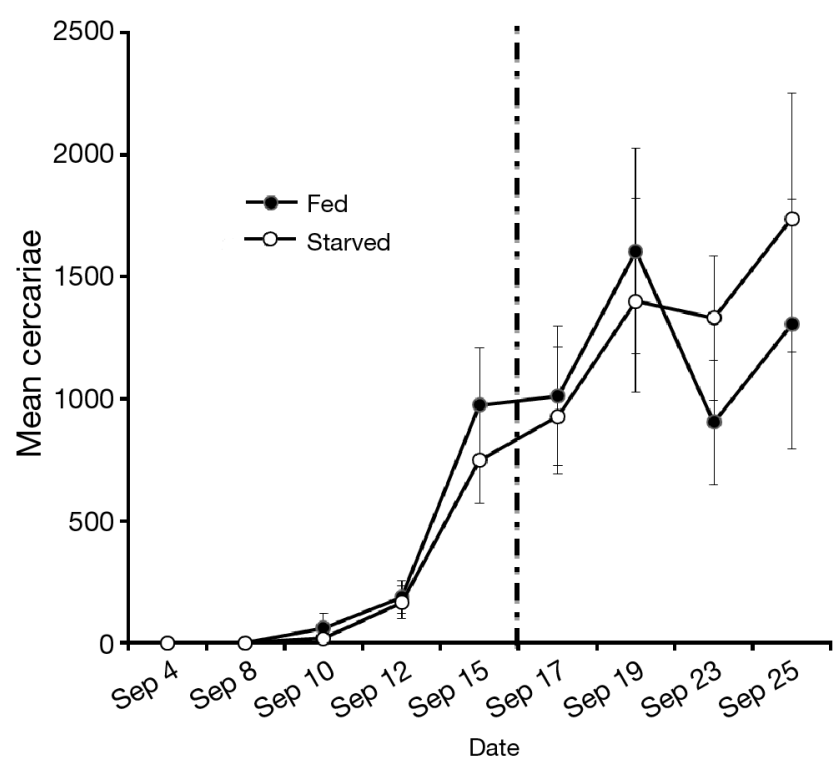

Fig. 3. Mean ( \pm SE) Echinostoma caproni cercarial output from host Biomphalaria glabrata snails before and after the onset of nutrient restriction in the snails. Dashed line represents the time point $38 \mathrm{~d}$ post exposure when snails allocated to the starved treatment experienced food restriction
41.97, $\mathrm{p}<0.001)$, time $\left(F_{2.9,23.4}=41.16, \mathrm{p}<0.001\right)$, and the diet $\times$ time interaction $\left(F_{2.9,23.4}=8.66, \mathrm{p}=0.001\right)$ were all significant. Again, the significant interaction term was driven by the fact that cercariae from starved snails stopped moving sooner than larvae shed from fed snails (Fig. 4B). In fact, after $15 \mathrm{~h}$ of observation, all of the cercariae shed from starved snails had stopped moving, whereas close to $50 \%$ of the cercariae from the fed hosts continued to move by the end of the experiment.

\section{DISCUSSION}

Although host nutrition is well recognized to play an important role in host and parasite life histories, the direction and magnitude of these responses can vary dramatically across systems (Logan et al. 2005, Beldomenico et al. 2008, Tadiri et al. 2013). In this study, we assessed the life history responses of Biomphalaria glabrata, an important host in the life cycle of human schistosomes, to both nutrient manipulation and infection by another trematode, Echinos-
Fed and starved snails used specifically for the cercarial experiments demonstrated no difference in parasite release $(p>0.05)$ on the final shedding day (47 DPE); however, swimming and general movement patterns varied significantly between the cercariae collected from starved vs. fed snails at that time. Repeated measures ANOVA demonstrated significant effects of diet $\left(F_{1,8}=12.73, \mathrm{p}=0.007\right)$, time $\left(F_{2.3,18.4}=\right.$ 68.36, $\mathrm{p}<0.001)$, and a diet $\times$ time interaction $\left(F_{2.3,18.4}=3.73, \mathrm{p}=0.039\right)$ on cercarial swimming. The proportion of swimming cercariae decreased over time, but decreased much more rapidly in cercariae from starved snails relative to those from fed snails (Fig. 4A). A similar pattern was observed for overall cercarial movement where diet $\left(F_{1,8}=\right.$

Fig. 4. Mean ( \pm SE) proportion of Echinostoma caproni cercariae that were (A) swimming or (B) moving after release from either starved or fed Biomphalaria glabrata snails. Cercariae were assessed hourly for $24 \mathrm{~h}$. Five cercariae snail $^{-1}$ were monitored, and 5 snails from each treatment group were assessed, resulting in a total of 50 cercariae monitored over the $24 \mathrm{~h}$ observation period

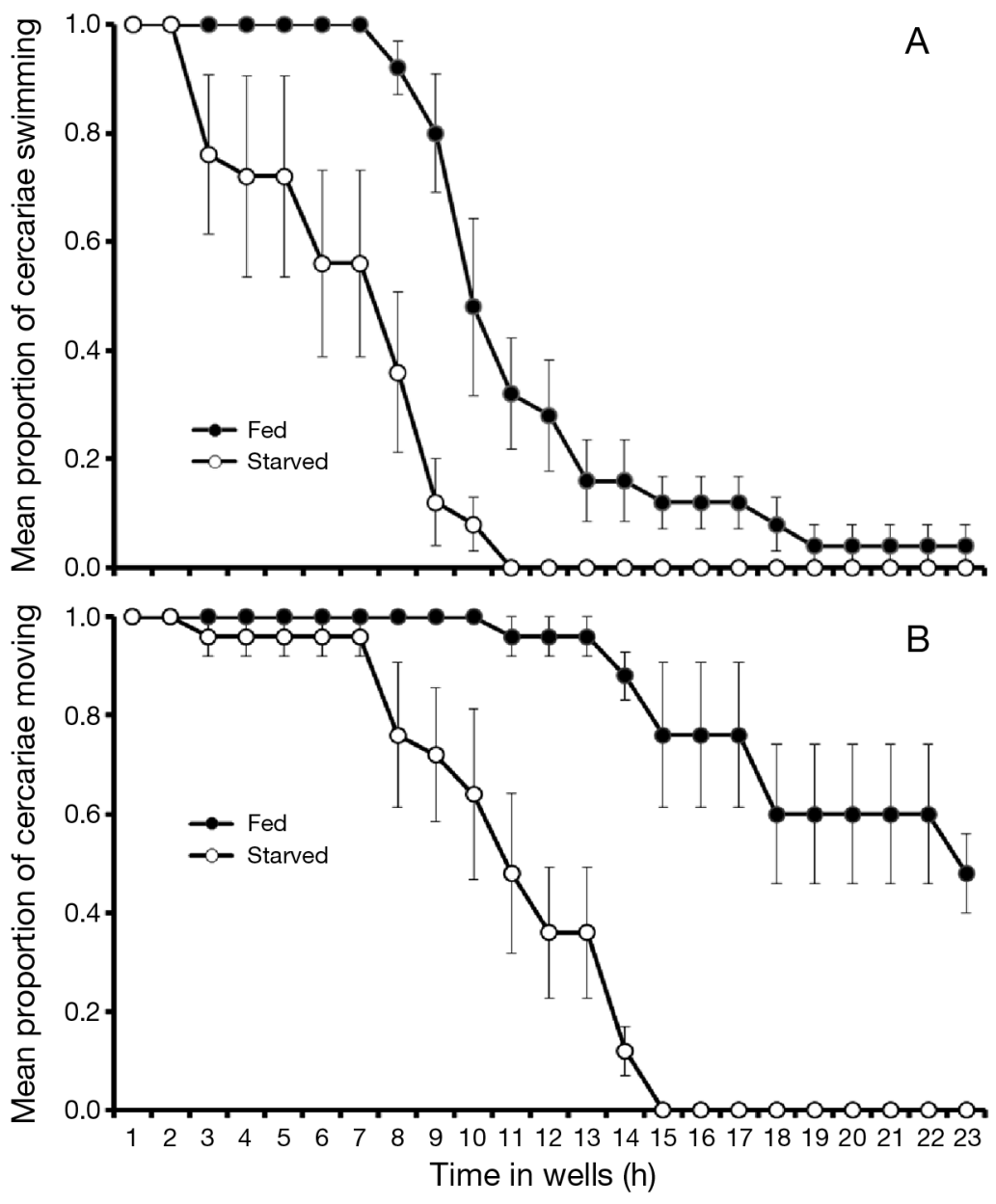


toma caproni. Furthermore, we monitored the life history responses and transmission potential of parasites from both fed and nutrient-deprived hosts.

In general, E. caproni infection substantially reduced B. glabrata egg output compared to uninfected hosts. Combining infection with nutrient restriction led to an immediate cessation of snail reproduction after the onset of starvation, whereas fed-infected snails continued to generate eggs over the remaining $2 \mathrm{wk}$. Parasitic sterilization is a wellrecognized phenomenon across snail-trematode systems (Sorensen \& Minchella 2001), including those involving echinostomes (Sandland \& Minchella 2003b). Although echinostome-infected snails eventually cease reproducing even under ad libitum diet regimes (Sandland \& Minchella 2003b, Sandland et al. 2007), results from this work suggest that adequate access to nutrients can extend the 'reproductive window' of infected hosts perhaps by prolonging gonadal maintenance (function) and/or extending the suppression of parasite exploitation through immunological mechanisms (such as hemocyte responses, lectin production, etc.) that may reduce nutrient uptake by rediae.

Host mortality was minimal in control snails throughout our study, whereas both fed and starved infected snails began to exhibit relatively high levels of mortality between Weeks 6 and 7. This pattern coincided with the onset of cercarial release from infected snails. Like many other echinostome species, E. caproni cercariae have the capacity to reinfect and encyst within the same infected host. Damage accrued via (1) cercarial migrations out of and back into the same host, (2) subsequent larval encystment in host organs such as the heart and kidney, and/or (3) host immunological responses to larval stages are factors that may have contributed to the high mortality seen in the infected B. glabrata in this study.

Nutrient restriction has been shown to intensify mortality in infected hosts (Pedersen \& Greives 2008, Beldomenico et al. 2008), including snails harboring trematodes (Krist et al. 2004). Researchers have also demonstrated that this pattern is not always consistent across host-parasite interactions (Jokela et al. 1999, 2005). In our study, control snails exhibited the same survival patterns regardless of whether they were fed or starved. Infected hosts, however, died more rapidly and exhibited greater overall mortality under resource restriction, supporting the idea that parasitism and nutritional stress can work in concert to exacerbate host pathology. Echinostome nutrient demand (Cheng \& Lee 1971) and/or host defensive costs (Sandland \& Minchella 2003a) combined with reduced carbohydrate pools in starved B. glabrata (Christie et al. 1974) may have depleted resources below the threshold required for snail survival within this treatment. Given the extent of parasitism and resource scarcity through time and across habitats (Logan et al. 2005), our results may provide a clearer picture of host life history responses to infection under natural circumstances. Together, this work along with past studies highlight the importance of considering nutrient availability when assessing the responses of individual hosts and host populations to parasitism (Beldomenico \& Begon 2010).

Effects of host nutrient restriction on parasite life histories have been investigated in several systems, often with variable results. For example, Krasnov et al. (2005) reported that fleas feeding on nutrientdeprived rodents produced more eggs that exhibited greater survival than fleas feeding on fed hosts. Conversely, Seppala et al. (2008) reported that starved, field-infected Lymnaea stagnalis released fewer Dipostomum spatheceum cercariae compared to snails that were fed ad libitum. Finally, Sandland \& Minchella (2003b) showed no differences in the numbers of $E$. revolutum cercariae shed from sizematched Stagnicola elodes snails maintained on either high- or low-quality diets. In our study, E. caproni cercariae were released in similar numbers regardless of host nutrient treatment, reflecting the results of Sandland \& Minchella (2003b). This pattern suggests that the quantity of cercariae produced by E. caproni, and possibly by echinostomes in general, may be a relatively inflexible trait (at least over the short term), even when the nutritional status of the host changes.

Although fed and starved B. glabrata shed similar quantities of E. caproni cercariae, larval quality was lower in parasites from starved versus fed snails. In parasites such as trematodes, carbohydrates (such as glycogen) play an important role in the lifespan and transmission success of cercariae (Lawson \& Wilson 1980, Fried et al. 1998, Karvonen et al. 2003), and past research suggests that they acquire these molecules from their snail hosts (Cheng \& Lee 1971, Christie et al. 1974, Pinheiro \& Amato 1994). However, carbohydrates, such as glycogen, can be substantially reduced in nutrient-deprived $B$. glabrata (Christie et al. 1974). If the starved B. glabrata in this study were carbohydrate depleted, E. caproni rediae and/or cercariae would have had fewer host resources to sequester and subsequently utilize for transmission. This may help to explain why larvae from nutrient-restricted hosts exhibited diminished 
swimming activity and reductions in overall movement. Given that we did not assess cercarial quality in snails prior to starvation, we cannot completely exclude the possibility that, by chance, snails allocated to the starved treatment produced lower-quality cercariae both before and after the onset of nutrient restriction. However, this possibility is unlikely given that the snails used in this study were of similar genetic background (strain) and were all maintained under the same laboratory colony conditions before the experiment and prior to the onset of starvation within the experiment (e.g. same light-dark cycle, feeding regime, water changes, etc.),

In other host-parasite systems, nutrient-mediated effects have been postulated to negatively influence parasite transmission and ultimately parasite populations (Logan et al. 2005). Reductions in cercarial activity along with the increases in snail mortality observed in our study suggest that $B$. glabrata nutritional status could indeed lead to a reduction in $E$. caproni transmission. However, factors such as cercarial specificity, second intermediate host densities, and environmental impediments to host contact must all be considered in association with larval quantity before the transmission consequences of host nutrient restriction can be fully elucidated in $E$. caproni.

Results from this study demonstrate that nutrient restriction in combination with infection can have important consequences not only for hosts, but also for the parasites themselves. Continued assessments of nutrient limitation across additional snail-trematode systems, including those with cercariae that infect non-molluscan species, should help to disentangle the contributions that particular larval stages (such as rediae and/or sporocysts) have on host pathology. Furthermore, extending current research to include estimates of infection at subsequent stages in the life cycle would provide a more definitive estimate of transmission success in E. caproni and in other trematode species infecting nutrientdeprived hosts. Incorporating these additional factors into future work should allow us to better resolve the role that host nutrient restriction plays in the persistence and magnitude of disease in host populations.

Acknowledgements. We thank Dr. Tom Platt (Saint Mary's College, Indiana) for providing us with E. caproni metacercariae. We also acknowledge Drs. M. Hoffman, D. Sewell, and B. Taylor for their support of and feedback on the project. This work was made possible via a Research, Service, Education and Leadership (RSEL) grant from the University of Wisconsin-La Crosse.

\section{LITERATURE CITED}

Beers K, Fried B, Fujino T, Sherma J (1995) Effects of diet on the lipid composition of the digestive gland-gonad complex of Biomphalaria glabrata (Gastropoda) infected with larval Echinostoma caproni (Trematoda). Comp Biochem Physiol B Biochem Mol Biol 110:729-737

Beldomenico PM, Begon M (2010) Disease spread, susceptibility and infection intensity: vicious circles? Trends Ecol Evol 25:21-27

Beldomenico PM, Telfer S, Gebert S, Lukomski L, Bennett M, Begon M (2008) Poor condition and infection: a vicious circle in natural populations. Proc Biol Sci 275: 1753-1759

* Bouchut A, Sautiere PE, Coustau C, Mitta G (2006) Compatibility in the Biomphalaria glabrata/Echinostoma caproni model: potential involvement of proteins from hemocytes revealed by a proteomic approach. Acta Trop 98:234-246

Brodersen G, Johns R, Lapointe R, Thumbi D, Thurston G, Lucarotti CJ, Quiring D (2012) Larval diet prior to and following virus ingestion influences the efficacy of two nucleopolyhedroviruses in whitemarked tussock moth (Orgyia leucostigma) caterpillars. Can Entomol 144: 447-457

Cheng TC, Lee FO (1971) Glucose levels in the mollusk Biomphalaria glabrata infected with Schistosoma mansoni. J Invertebr Pathol 18:395-399

Christie JD, Foster WB, Stauber LA (1974) The effect of parasitism and starvation on carbohydrate reserves of Biomphalaria glabrata. J Invertebr Pathol 23:55-62

* Civitello DJ, Penczykowski RM, Smith AN, Shocket MS, Duffy MA, Hall SR (2015) Resources, key traits and the size of fungal epidemics in Daphnia populations. J Anim Ecol 84:1010-1017

* Dlugosz EM, Downs CJ, Khokhlova IS, Degen AA, Krasnov BR (2014) Host reproductive status and reproductive performance of a parasite: offspring quality and trade-offs in a flea parasitic on a rodent. Parasitology 141:914-924

* Ebert D, Carius HJ, Little T, Decaestecker E (2004) The evolution of virulence when parasites cause host castration and gigantism. Am Nat 164:S19-S32

F Forbes KM, Henttonen H, Hirvelä-Koski V, Kipar A, Mappes T, Stuart P, Huitu O (2015) Food provisioning alters infection dynamics in populations of a wild rodent. Proc Biol Sci 282:20151939

Fried B, Eyster LS, Pechenik JA (1998) Histochemical glycogen and neutral lipid in Echinostoma trivolvis cercariae and effects of exogenous glucose on cercarial longevity. J Helminthol 72:83-85

* Gerard G (1996) Energy constraint exerted by the parasite Schistosoma mansoni on the locomotion of its snail host, Biomphalaria glabrata. Can J Zool 74:594-598

Jokela J, Lively CM, Taskinen J, Peters AD (1999) Effect of starvation on parasite-induced mortality in a freshwater snail (Potamopyrgus antipodarum). Oecologia 119: 320-325

Jokela J, Taskinen J, Mutikainen P, Kopp K (2005) Virulence of parasites in hosts under environmental stress: experiments with anoxia and starvation. Oikos 108:156-164

Karvonen A, Paukku S, Valtonen ET, Hudson PJ (2003) Transmission, infectivity and survival of Diplostomum spathaceum cercariae. Parasitology 127:217-224

Koski KG, Su Z, Scott ME (1999) Energy deficits suppress both systemic and gut immunity during infection. Biochem Biophys Res Commun 264:796-801 
Krasnov BR, Khokhlova IS, Arakelyan MS, Degen AA (2005) Is a starving host tastier? Reproduction in fleas parasitizing food-limited rodents. Funct Ecol 19:625-631

Krist AC, Jokela J, Wiehn J, Lively CM (2004) Effects of host condition on susceptibility to infection, parasite developmental rate, and parasite transmission in a snail-trematode interaction. J Evol Biol 17:33-40

Lawson JR, Wilson RA (1980) The survival of the cercariae of Schistosoma mansoni in relation to water temperature and glycogen utilization. Parasitology 81:337-348

Lefèvre $\mathrm{T}$, Vantaux A, Dabiré KR, Mouline $\mathrm{K}$, Cohuet A (2013) Non-genetic determinants of mosquito competence for malaria parasites. PLOS Pathog 9:e1003365

Logan A, Ruiz-González MX, Brown MJ (2005) The impact of host starvation on parasite development and population dynamics in an intestinal trypanosome parasite of bumble bees. Parasitology 130:637-642

Muturi EJ, Kim CH, Alto BW, Berenbaum MR, Schuler MA (2011) Larval environmental stress alters Aedes aegypti competence for Sindbis virus. Trop Med Int Health 16: 955-964

Pedersen AB, Greives TJ (2008) The interaction of parasites and resources cause crashes in a wild mouse population. J Anim Ecol 77:370-377

Pinheiro J, Amato JF (1994) Eurytrema coelomaticum (Digenea: Dicrocoeliidae): the effect of infection on carbohydrate contents of this intermediate snail host, Bradybaena similaris (Gastropoda, Xanthonychidae). Mem Inst Oswaldo Cruz 89:407-410

Rivero A, Agnew P, Bedhomme S, Sidobre C, Michalakis Y (2007) Resource depletion in Aedes aegypti mosquitoes infected by the microsporidia Vavrala cullcis. Parasitology 134:1355-1362

Rondelaud D, Denève C, Belfaiza M, Mekroud A, Abrous M, Moncef M, Dreyfuss G (2004) Variability in the preva-

Editorial responsibility: Stephen Feist, Weymouth, UK lence of infection and cercarial production in Galba truncatula raised on a high-quality diet. Parasitol Res 92: 242-245

Rosemond AD, Mulholland PJ, Brawley SH (2000) Seasonally shifting limitation of stream periphyton: response of algal populations and assemblage biomass and productivity to variation in light, nutrients, and herbivores. Can J Fish Aquat Sci 57:66-75

Sandland GJ, Minchella DJ (2003a) Costs of immune defense: an enigma wrapped in an environmental cloak? Trends Parasitol 19:571-574

* Sandland GJ, Minchella DJ (2003b) Effects of diet and Echinostoma revolutum infection on energy allocation patterns in juvenile Lymnaea elodes snails. Oecologia 134: 479-486

Sandland GJ, Rodgers JK, Minchella DJ (2007) Interspecific antagonism and virulence in hosts exposed to two parasite species. J Invertebr Pathol 96:43-47

Seppala O, Lilijeroos K, Karvonen A, Jokela J (2008) Host condition as a constraint for parasite reproduction. Oikos 117:749-753

Sorensen RE, Minchella DJ (2001) Snail-trematode life history interactions: past trends and future directions. Parasitology 123:S3-S18

*Tadiri CP, Dargent F, Scott ME (2013) Relative host body condition and food availability influence epidemic dynamics: a Poecilia reticulata-Gyrodactylus turnbulli host-parasite model. Parasitology 140:343-351

Toledo R, Muñoz-Antoli C, Fried B (2007) The use of echinostomes to study host-parasite relationships between larval trematodes and invertebrate and cold-blooded vertebrate hosts. Parasitol Res 100:1177-1185

Tschirren B, Bischoff LL, Saladin V, Richner H (2007) Host condition and host immunity affects parasite fitness in a bird-ectoparasite system. Funct Ecol 21:372-378

Submitted: January 23, 2017; Accepted: August 21, 2017

Proofs received from author(s): September 22, 2017 